\title{
BUBBLE DEPARTURE FROM METAL-GRAPHITE COMPOSITE SURFACES AND ITS EFFECTS ON POOL BOILING HEAT TRANSFER
}

\author{
David F. Chao, ${ }^{1}$ John M. Sankovic, ${ }^{1}$ Brian J. Motil, ${ }^{1}$
}

W-J. Yang, ${ }^{2}$ E Nengli Zhang ${ }^{3 *}$

${ }^{1}$ NASA Glenn Research Center, Cleveland, OH 44135

${ }^{2}$ The University of Michigan, Ann Arbor, MI 48109

${ }^{3}$ Ohio Aerospace Institute at NASA Glenn Research Center, Cleveland, $\mathrm{OH} 44135$

*Address all correspondence to Nengli Zhang E-mail: nzhang@grc.nasa.gov

The formation and growth processes of a bubble in the vicinity of graphite micro-fiber tips on metal-graphite composite boiling surfaces and their effects on boiling behavior are investigated. It is discovered that a large number of micro bubbles are formed first at the micro scratches and cavities on the metal matrix in pool boiling. By virtue of the non-wetting property of graphite, once the growing micro bubbles touch the graphite tips, the micro bubbles are sucked by the tips and merged into larger micro bubbles sitting on the end of the tips. The micro bubbles grow rapidly and coalesce to form macro bubbles, each spanning several tips. The necking process of a detaching macro bubble is analyzed. It is revealed that a liquid jet is produced by sudden break-off of the bubble throat. The composite surfaces not only have higher temperatures in micro- and macrolayers but also make higher frequency of the bubble departure, which increase the average heat fluxes in both the bubble growth stage and in the bubble departure period. Based on these analyses, the enhancement mechanism of pool boiling heat transfer on composite surfaces is clearly revealed.

KEY WORDS: micro bubbles, macro bubbles, macro bubble departure, bubble necking, liquid jet, boiling heat transfer enhancement

\section{INTRODUCTION}

It is well known that nucleate boiling is an extremely effective mode of heat transfer. Although over the years researchers have studied the boiling process theoretically and experimentally, there are no theories in the literature that precisely 


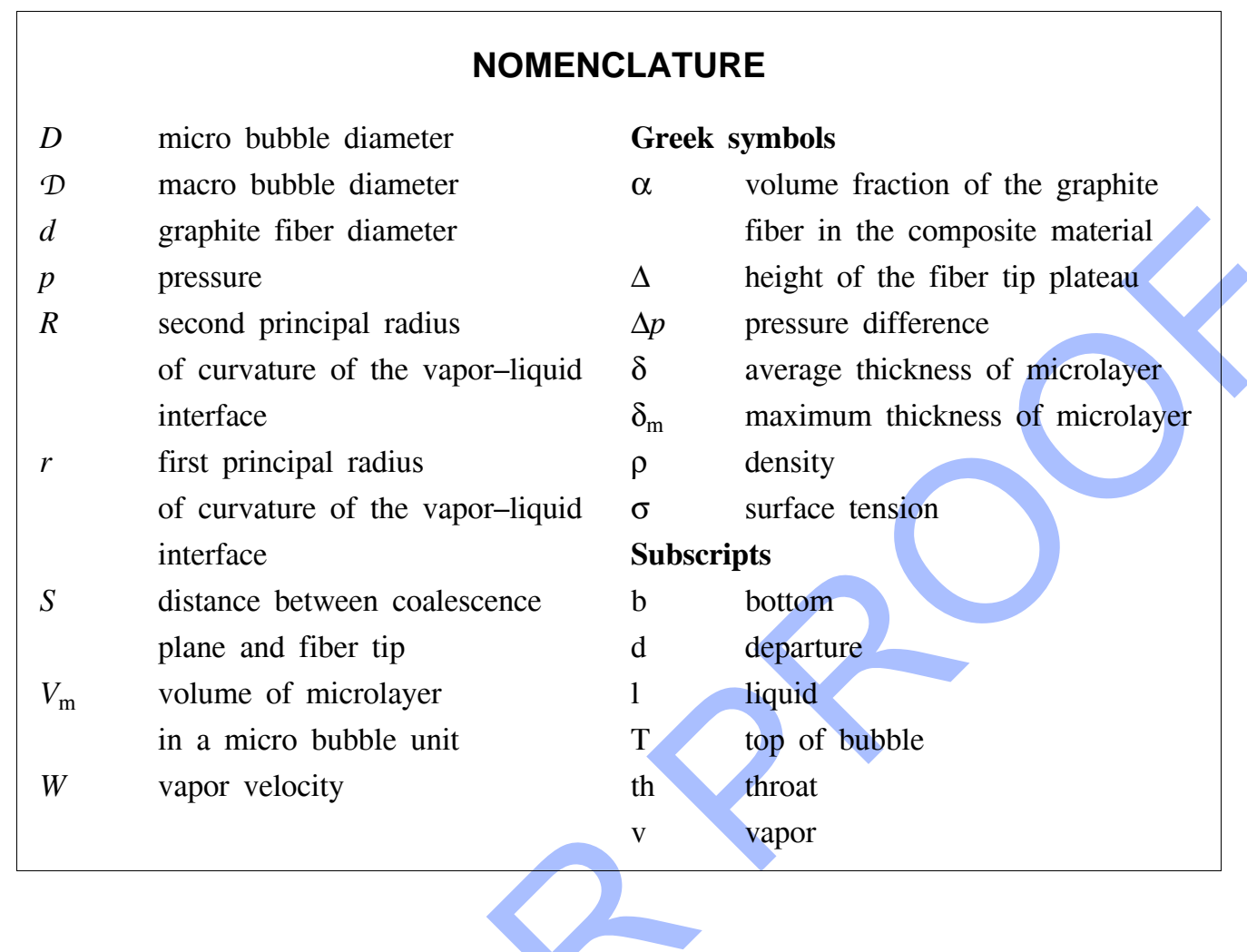

explain the underlying heat transfer mechanisms. However, an accepted theory is that bubble nucleation, growth, and departure control the heat and mass transport rate. The basic understanding of boiling phenomena on plain surfaces brought about concepts to modify the process through changing properties of the working fluid and/or the boiling surface to increase the boiling heat transfer coefficient. A new passive technique, called metal-graphite composite surfaces, was presented and studied by Yang et al. (1991). Since then a series of investigations have been performed to determine the enhancement effects of composite surfaces on boiling heat transfer and their mechanism (see, e.g., Zhang et al., 1992; Yang, 1995; Liang, 1997). The practical importance of the new enhancement technique applying to Multi-Chip Modules (MCM) was discussed by Vrable (2001). Chao et al. (2004) conducted experiments on nucleate pool boiling both in a highly wetting liquid, Freon-113, and a moderately wetting fluid, water, on $\mathrm{Cu}-\mathrm{Gr}$ composite surfaces with different fiber volume fractions. The experimental results have shown that significant enhancement in pool boiling heat transfer performance in both highly wetting liquids and moderate wetting liquids occurs on $\mathrm{Cu}-\mathrm{Gr}$ composite surfaces.

The enhancement mechanisms of boiling on the composite surfaces are believed to be related to: 1) the higher thermal conductivity of micro-graphite fibers, 2) a large number of embryo cavities of appropriate size, and 3) the poorly wetted 
property of graphite fiber tips (Liang and Yang, 1998[Q1]). In order to clarify the physics of the enhancement, it is important to have an understanding of the processes of bubble formation, growth and departure and their effects on the boiling heat transfer.

For common solid surfaces, numerous models have been developed to predict bubble detachment diameters in pool boiling and were reviewed by Zeng et al. (1993). Many experiments have shown that bubble detachment had been implemented by a necking process (see van Stralen et al., 1975; Nordmann and Mayinger, 1981; Mitrovic, 1997, 1998; Mori and Baines, 2001). Mitrovic (1997, 1998) analyzed the movement of three-phase-line (TPL) and the formation of bubble neck during bubble growth. He conjectured that evaporation at TPL tended to create a convex interface, leading to Laplace-pressure, which pushed the liquid radially outward to form the bubble neck. Kandlikar and Steinke (2002) suggested that an intense evaporation at the TPL exerted a vapor recoil force pushing the interface into the liquid and consequently, a convex vapor-liquid interface was formed and developed into the bubble neck. The bubble necking and brake-off processes were described by Mitrovic (1997) and Mori and Baines (2001), but the dynamic process has not been properly analyzed. Recently, Zhang et al. (2006) analyzed the detail dynamic process of the bubble growth on a cavity. The bubble necking and departure were mathematically described, and subsequently, a determination method of the bubble departure diameter was presented. It certainly is of interest to understand the bubble behavior on the composite surfaces and its effects on the boiling heat transfer. This paper analyzes the micro- and macro-bubble formation, growth on a metal-graphite composite surface, and the macro bubble departure dynamic processes from the surface. The mechanism of a liquid jet formation after the macro bubble departure has been revealed. The effects of the bubble departure on the boiling heat transfer are discussed.

\section{FORMATION AND GROWTH OF MICRO BUBBLES}

Different from common metal surfaces, metal-graphite composite surfaces each have their unique geometric and physical characteristics. The polished composite surfaces are smooth in macro scale but rough in micro scale. Yang (1995) investigated the surface structure of $\mathrm{Cu}-\mathrm{Gr}$ composite surfaces through a scanning electronic microscope (SEM). Direct top-view photomicrographs of a $\mathrm{Cu}-\mathrm{Gr}$ composite specimen with a volume fraction of $50 \%$ graphite-fiber and a fiber diameter of 8 $10 \mu \mathrm{m}$ clearly show that the graphite fibers are embedded in the copper matrix perfectly without any gap or void. The photomicrographs also show that the fiber tips are rather smooth while the copper matrix surface is filled with a large number of micro size low-lying trenches and intermingled narrow grooves. A slightly tilted top-view of the composite surface shows that the fiber tips bulge out of the copper matrix, surviving the polishing due to their pliability and tenacity, and form individual plateaus distributing evenly on the composite surfaces.

Volume 17, Number 4, 2010 


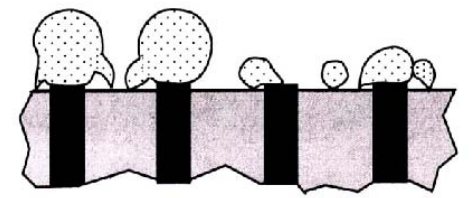

a)

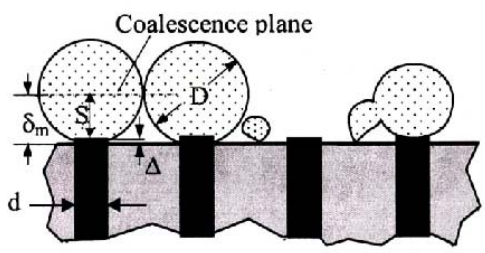

b)

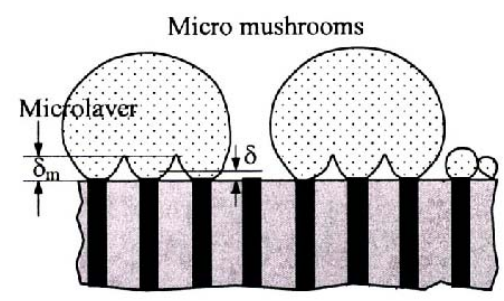

c)

FIG. 1: Initiation and growth of micro bubbles: a) initiation and migration of micro bubbles; b) growing of micro bubbles firmly sitting on the fiber tips; c) growth of coalesced micro bubbles and formation of micro mushrooms

Contrary to intuition, the preliminary experimental observations have found that the micro bubbles originate from the valleys between the fiber tip plateaus instead of the fiber tips. It is obviously attributed to the fact that plenty of potential nucleate sites are created by the micro-sized trenches and intermingled narrow grooves on the copper matrix surface. On the other hand, although the fiber tips may have higher temperatures than the copper matrix surface, the relative smooth surface of the fiber tip plateaus hardly provide initial nucleate sites.

Once the growing micro bubbles touch the fiber tips, the micro bubbles tend to migrate to the tips from the copper matrix and firmly sit on the tips for the poor wetting characteristic of the graphite and a large surface tension at the TPL. As the micro bubbles sitting on the fiber tips grow, the newly emerging embryo bubbles on the copper matrix are swallowed by the micro bubbles at contact, as shown in Fig. 1a. Both the rapid evaporation due to the higher temperature of the graphite fiber tips and the swallowing of the emerging embryo micro bubbles speed up the growth of the micro bubbles on the fiber tips. The continually growing micro bubbles inevitably coalesce with each other, as shown in Fig. 1b. The coalesced micro bubbles rapidly grow into micro mushrooms with their own microlayers forming a liquid network connected with bulk liquid under the coalesced micro bubbles, as shown in Fig. 1c.

As analyzed by Zhang et al. (1992), the micro bubbles coalesce at their maximum cross section. By reference to Fig. 1b, the micro bubble diameter at coales- 
cence, $D$, is related to the fiber diameter, $d$, and the volume fraction of the graphite fiber in the composite material, $\alpha$ which is equal to the area fraction of the fiber tips in the composite surface, by the following equation:

$$
\pi d^{2} / 4=\alpha D^{2} .
$$

The height of the coalescence plane of the micro bubbles is regarded as the maximum thickness of the microlayer $\delta_{\mathrm{m}}$. It can be seen from Fig. $1 \mathrm{~b}$ that $\delta_{\mathrm{m}}=$ $\Delta+S$, where $\Delta$ is the height of the fiber tip plateau, estimated at approximately $0.3 \mu \mathrm{m}, S$ is the distance between the coalescence plane and fiber tip, equaling $\sqrt{(D / 2)^{2}-(d / 2)^{2}}=d \sqrt{\pi /(4 \alpha)-1} / 2$.

Therefore,

$$
\delta_{m}=\frac{d}{2} \sqrt{\frac{\pi}{4 \alpha}-1}+\Delta
$$

Based on simple geometry, the volume of the microlayer in a micro bubble unit can be calculated by

$$
V_{m}=D^{2} \delta_{m}-\frac{1}{6} \pi S\left[\frac{3}{4} D^{2}+\frac{3}{4} d^{2}+S^{2}\right]-\frac{\pi d^{2}}{4} \Delta .
$$

Idealizing the liquid volume as a column of constant cross section, the average thickness of microlayer, $\delta$, can be derived by $V_{\mathrm{m}} /\left(D^{2}-\pi d^{2} / 4\right)$. As a result, the following expression can be obtained:

$$
\delta=\left[\frac{1-\pi / 6-\alpha / 3}{2(1-\alpha)} \sqrt{\frac{\pi}{4 \alpha}-1}\right] d+\Delta .
$$

Obviously, both the maximum and average thicknesses of the microlayer are determined by the volume fraction of graphite fiber in the composite $\alpha$ and the fiber diameter $d$. As mentioned above, $d=8-10 \mu \mathrm{m}$. Simple calculation results for the maximum and average thicknesses of the microlayer at $d=9 \mu \mathrm{m}$ are listed in Table 1.

In boiling heat transfer, microlayers play an important role. Thermal energy provided by the composite surface is first conducted through the microlayers and then

TABLE 1: Microlayer thicknesses at different values of $\alpha$

\begin{tabular}{|l|c|c|c|c|}
\hline$\alpha$ & 0.1 & 0.25 & 0.5 & 0.75 \\
\hline$\delta_{\mathrm{m}}, \mu \mathrm{m}$ & 12.08 & 6.89 & 3.70 & 1.28 \\
\hline$\delta, \mu \mathrm{m}$ & 6.10 & 3.75 & 2.41 & 1.19 \\
\hline
\end{tabular}


delivered into the micro mushrooms as latent heat by evaporation at the microlayer surface. Because the microlayers are connected directly with the bulk liquid through a liquid network, the liquid evaporating off the microlayers can be replenished immediately. It is the ample feed of liquid into the microlayers that enables the composite surfaces to have lower superheats at given boiling heat fluxes, i.e., enhancement of boiling heat transfer. This is only a sweeping statement; the direct actions of the enhancement are actually executed by the macro bubble departure.

\section{FORMATION OF A MACRO BUBBLE}

The continually growing micro mushrooms inevitably coalesce and merge with each other to form a larger micro vapor mass, which is a macro bubble embryo, as shown in Fig. 2. When the bubble embryo is formed from several micro mushrooms, a macrolayer is created under the macro bubble embryo, connecting with the microlayers and bulk liquid through a liquid network. As analyzed by Mitrovic (1997, 1998) and Kandlikar and Steinke (2002), at the beginning of the bubble formation, the TPL of the bubble moves outwards to form a convex vapor-liquid interface at the bubble bottom, and subsequently a bubble neck is created. The same process also occurs in the growth of a macro bubble embryo on the composite surfaces. As the macro bubble embryo grows, the TPL of the macrolayer moves towards the liquid under the combination of a high evaporation rate and a capillary-pressure difference. Zhang et al. (2006) analyzed this kind of TPL moving process in detail. In the case of a macro bubble embryo formed on the metalgraphite composite surfaces, the moving TPL would stop at the next circles consisting of the corresponding neighbor graphite fiber tips for the poor wettability of the graphite fiber. Consequently, convex vapor-liquid interfaces are created at the macro bubble embryo bottom at both the inner and the outer TPLs, as shown

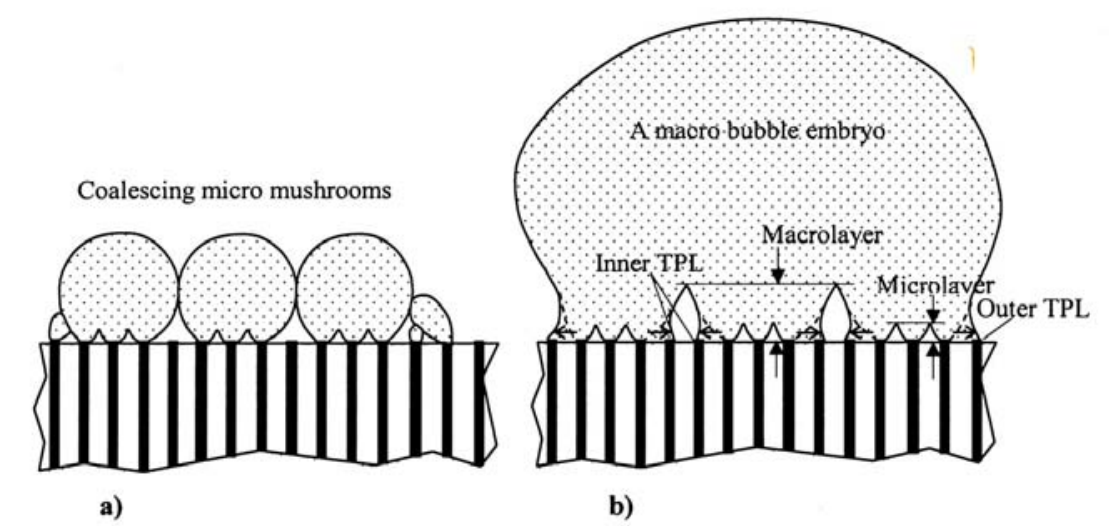

FIG. 2: Formation of a macro mushroom from coalesced micro mushrooms: a) coalescence of micro mushrooms; b) formation of a macro bubble embryo 


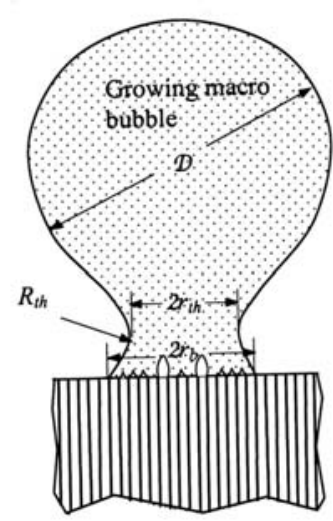

FIG. 3: Formation of a macro bubble

in Fig. 2b. The macro bubble embryo rapidly increases and finally forms a macro bubble with a neck throat, as shown in Fig. 3.

\section{DEPARTURE OF A MACRO BUBBLE}

The strong evaporation of liquid from the micro- and macrolayers is firmly supported by the ample liquid supply through the liquid network at the bubble bottom, and creates larger and larger negative pressures at the throat, i.e., $\Delta p_{\text {th }}=p_{\mathrm{v} \text {,th }}$ $-p_{1, \text { th }}<0$, where $p_{\mathrm{v}, \text { th }}$ and $p_{1, \text { th }}$ are the static pressures of vapor side and liquid side at the throat, respectively.

It should be noted that the static pressure difference varies along the bubble interface, as shown in Fig. 3. The static pressure difference at the top of a departing bubble, $\Delta P_{\mathrm{T}}$, can be expressed as $4 \sigma / \mathcal{D}_{\mathrm{d}}$, where $\sigma$ is the surface tension, $\mathcal{D}_{\mathrm{d}}$ is the bubble departure diameter, while the static pressure difference at the throat $\Delta p_{\text {th }}$ is estimated by

$$
\Delta p_{t h}=\sigma / r_{t h}-\sigma / R_{t h}-\rho_{v} W_{t h}^{2} / 2
$$

where $r_{\text {th }}$ and $R_{\text {th }}$ are the first and second principal curvature radius of the vaporliquid interface at throat, respectively, $\rho_{\mathrm{v}}$ is the density of vapor, $W_{\mathrm{th}}$ is the vapor velocity at throat. It should be pointed out that some researchers wrongly described the pressure difference at the throat for missing the last term in Eq. (5), which results in an unreasonable verdict: the pressure difference at the throat would always be positive and consequently no necking process would occur. It can be seen that the first two terms in Eq. (5) always give positive as soon as $R_{\text {th }}$ $>r_{\text {th }}$, to say nothing of the small values of $r_{\text {th }}$ with a larger $R_{\text {th }}$. Actually, it is the higher and higher velocity of vapor passing through the throat creates the

Volume 17, Number 4, 2010 


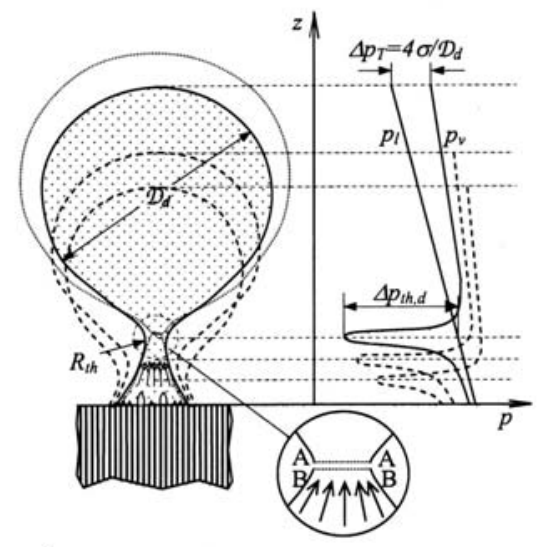

a)

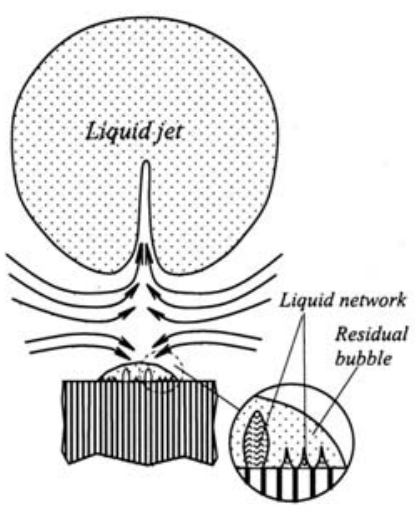

b)

FIG. 4: Growth, necking, and departure of a macro bubble: a) necking process; b) liquid jet after the macro bubble departure

larger and larger negative pressure at the throat, as indicated mathematically by Eq. (5). It can also be concluded that a detaching bubble will never depart with zero neck diameter, i.e., $r_{\text {th }} \neq 0$, otherwise, $\Delta p_{\text {th }} \rightarrow \infty$. In other words, along with the detaching bubble growth, the throat diameter of the bubble quickly contracts to a finite value when the buoyancy exceeds the surface tension force at the throat and consequently the neck breaks off suddenly. At this time, the major portion of the bubble above the neck departs with a singular circle line A-A, as shown in Fig. $4 \mathrm{a}$, where a considerable large pressure difference $\Delta p_{\text {th }}$ occurs. This is the reason that a liquid jet occurs in the departure bubble. Of course, the corresponding singular line $\mathrm{B}-\mathrm{B}$ is formed at the residual vapor portion sitting on the composite surface but the strong evaporation from the liquid network at the residual bubble bottom withstands the action of the sudden change of $\Delta p_{\text {th }}$, and at the same time rapidly fosters and develops a new bubble. Due to the bubble break-off at the throat suddenly occurs in a finite diameter of the neck, a huge momentum flow creates a liquid jet dashing into the bubble. It is the liquid jet that produces a violent wake of the departed bubble and induces a strong convection flow on the boiling surface to refresh the liquid network at the residual bubble, which supports the liquid supply for the new bubble growth, as depicted in Fig. 4b.

\section{ENHANCEMENT OF BOILING HEAT TRANSFER}

It is because of the unique structure of metal-graphite composite surfaces that the strong evaporation in macro bubbles is supported by the ample liquid supply through the liquid network connecting the macrolayers and microlayers to the bulk liquid. Consequently, a much higher heat flux can be reached, especially when the 


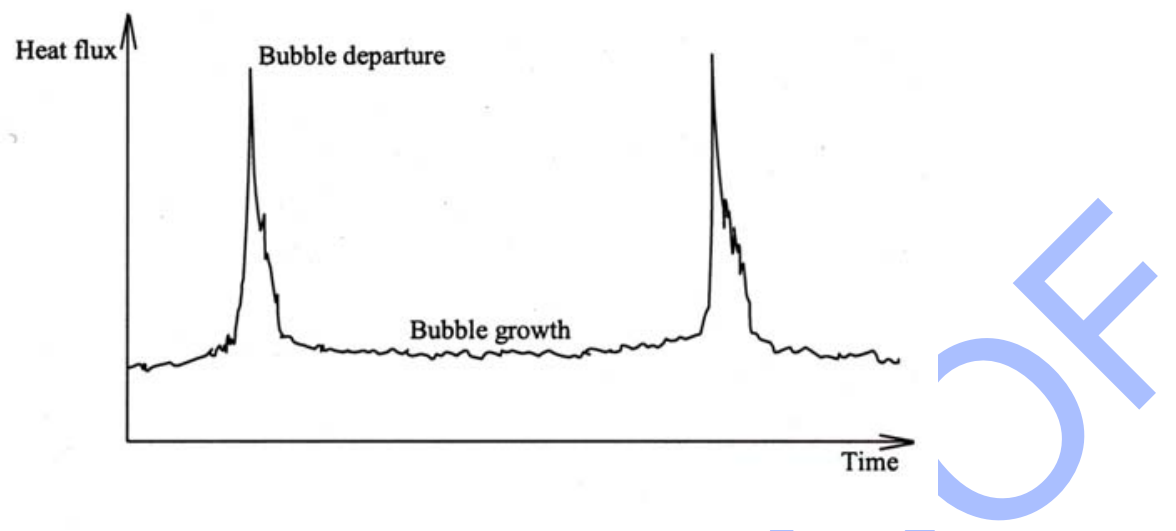

FIG. 5: Heat flux variation during a bubble life cycle

bubbles depart. As experimental demonstration by Chen and Chung (2002), a heat flux pulse takes place at the bubble departure while a lower heat flux period occurs in the bubble growth stage, as qualitatively shown in Fig. 5. The experiments using FC-72 on platinum surface made by Chen and Chung clearly show that the highest value of the heat flux pulse is about three times the heat flux in the bubble growth stage $\left(90 \mathrm{~W} / \mathrm{cm}^{2}\right.$ vs. $\left.30 \mathrm{~W} / \mathrm{cm}^{2}\right)$. Usually, higher temperatures of the micro- and macrolayers will increase the average heat flux for the larger conduction and higher evaporation rate in the micro- and macrolayers. Another efficient approach to increase heat flux is to increase the bubble departure frequency. It is easy to understand that higher frequency of bubble departure results in more heat flux pulses per unit time for the shorter bubble life cycle, and consequently, the average heat flux is increased. It is the metal-graphite composite surfaces that not only have higher temperature in micro- and macrolayers but also make higher frequency of the bubble departure, and therefore, average heat fluxes in both the bubble growth stage and the bubble departure period are increased. Based on the force balance at the neck of a departing bubble, Chao et al. (2004) have theoretically and experimentally demonstrated that higher vapor velocity produced by a larger local heat flux of boiling on the composite surface enables the bubbles to depart at smaller departure diameters, and consequently, results in an increased bubble departure frequency. This enhancement mechanism can successfully explain the experimental results of the enhanced boiling heat transfer both for F-113 and water (Yang et al., 1991; Chao et al., 2004).

\section{CONCLUSIONS}

In pool boiling on metal-graphite composite surfaces, micro bubbles originate first from the valleys between the fiber tip plateaus instead of the fiber tips on the graphite tips, and then migrate to the tips. The growing micro bubbles sitting on

Volume 17, Number 4, 2010 
the fiber tips coalesce and merge with each other to produce macro bubble embryos with a liquid network consisting of micro- and macro-layers. An important parameter, the microlayer thickness is analyzed and formulated.

It is the unique structure of metal-graphite composite surfaces that creates a liquid network connected to bulk liquid, from whom an ample supply of liquid firmly supports the strong evaporation at the surfaces of micro- and macrolayers and fosters and develops macro bubbles. During the formation process of a macro bubble, a convex-concave interface is created at the bubble bottom under the combination of a high evaporation rate and the capillary-pressure difference, and then develops to a neck with a decreasing throat diameter. It is the strong evaporation that produces increasing vapor velocities and creates a very high negative pressure at the bubble throat, which accelerates the reduction of the throat diameter and finally leads the macro bubble departure for the surface tension force failing to support the bubble against the buoyancy.

A liquid jet is created immediately after the departure of the macro bubble by a huge momentum flow due to the bubble throat break-off suddenly. The violent wake of the bubble created by the liquid jet induces a strong convection flow on the boiling surface to refresh the liquid network at the residual bubble bottom, which fosters the new bubble. It is the composite surfaces that not only have higher temperature in the micro- and macrolayers but also make higher frequency of the bubble departure, and consequently, increase the average heat fluxes both in the bubble growth stage and in the bubble departure period. In this way, the boiling heat transfer is enhanced.

\section{REFERENCES}

Chao, D. F., Zhang, N., and Yang, W. J., Nucleate pool boiling on copper-graphite composite surfaces and its enhancement mechanism, J. Thermophys. Heat Transfer, vol. 18, no. 2, pp. 236$242,2004$.

Chen, T. and Chung, J. N., Coalescence of bubbles in nucleate boiling on microheaters, Int. J. Heat Mass Transfer, vol. 45, no. 11, pp. 2329-2341, 2002.

Kandlikar, S. G. and Steinke, M. E., Contact angles and interface behavior during rapid evaporation of liquid on a heated surface, Int. J. Heat Mass Transfer, vol. 45, no. 18, pp. 3771-3780, 2002.

Liang, H. S., Nucleate Pool Boiling on Micro-Graphite-Fiber Composite Surfaces with Applications in Micro-Electronic Cooling, Ph.D, The University of Michigan, 1997.

Mitrovic, J., Formation of a liquid jet after detachment of a vapour bubble, Int. J. Heat Mass Transfer, vol. 40, no. 18, pp. 4309-4317, 1997.

Mitrovic, J., The flow and heat transfer in the wedge-shaped liquid film formed during the growth of a vapour bubble, Int. J. Heat Mass Transfer, vol. 41, no. 12, pp. 1771-1785, 1998.

Mori, B. K. and Baines, W. D., Bubble departure from cavities, Int. J. Heat Mass Transfer, vol. 44, no. 4, pp. 771-783, 2001.

Nordmann, D. and Mayinger, F., Temperatur, Druck und Wärmetransport in der umgebung kondensierender Blasen, VDI-Forschungsheft Nr. 605, 1981. 
van Stralen, S. J. D., Cole, R., Sluyter, W. M., and Sohal, M. S., Bubble growth rates in nucleate boiling of water at subatmospheric pressures, Int. J. Heat Mass Transfer, vol. 18, no. 5, pp. 655669, 1975.

Vrable, D. L., Composite material technology to enhance boiling heat transfer performance, Int. J. Transport Phenomena, vol. 3, no. 4, pp. 395-405, 2001.

Yang, G. W., Micro- and Macro-Phenomena in Nucleate Pool Boiling on Graphite-Copper Composite Materials, Ph.D, The University of Michigan, 1995.

Yang, W. J., Takizawa, H., and Varable, D., Augmented on copper-graphite composite surface, Int. J. Heat Mass Transfer, vol. 34, no. 11, pp. 2751-2758, 1991.

Zeng, L. Z., Klausner, J. F., and Mei, R., A unified model for the prediction of bubble detachment diameters in boiling systems-I. Pool boiling, Int. J. Heat Mass Transfer, vol. 36, no. 9, pp. 22612270, 1993.

Zhang, N., Chao, D. F., and Sankovic, J. M., Two basic modes of bubble growth and determination of departure diameters in pool boiling, AIAA Meeting Papers on Disc [CD-ROM], Section 142TP-10, 983 Reno, NV, 2006.

Zhang, N., Yang, W. J., and Yang, G. W., Two-tier model for nucleate pool boiling on micro configured composite surfaces, Int. Comm. Heat Mass Transfer, vol. 19, no. 6, pp. 767-779, 1992.

\section{[Q1] AU: This reference is not cited in the list of references. Please add}

Volume 17, Number 4, 2010 